\title{
Establishment and characterization of novel human oral squamous cell carcinoma cell lines from advanced-stage tumors of buccal mucosa
}

\author{
NILESH P. GAWAS ${ }^{1 *}$, SUSHANT S. NAVARANGE ${ }^{1,2^{*}}$, GOPAL L. CHOVATIYA $^{1,2}$, \\ PANKAJ CHATURVEDI $^{3}$ and SANJEEV K. WAGHMARE ${ }^{1,2}$
}

\author{
${ }^{1}$ Stem Cell Biology Group, Waghmare Lab, Cancer Research Institute, Advanced Centre for Treatment Research \\ and Education in Cancer (ACTREC), Tata Memorial Centre, Navi Mumbai 410210; \\ ${ }^{2}$ Homi Bhabha National Institute, Training School Complex, Anushakti Nagar, Mumbai 400085; \\ ${ }^{3}$ Department of Head and Neck Oncology, Tata Memorial Hospital, Mumbai 400012, India
}

Received May 14, 2018; Accepted November 29, 2018

DOI: $10.3892 /$ or.2019.7003

\begin{abstract}
Oral squamous cell carcinoma (OSCC) is a leading cause of mortality in India owing to the high percentage of tobacco chewers, smokers and alcohol consumption. OSCC is highly heterogeneous in nature; therefore poses a challenge in the treatment of the patient. To better understand the heterogeneity of the tumors, an in vitro cell line model is required. However, the efficiency of establishing cell lines from the oral tumors is low. In the present study, three novel cell lines, namely ACOSC 3 , ACOSC4, and ACOSC16, were isolated and characterized from advanced-stage treatment-naive OSCCs originating from the buccal mucosa. The three cell lines exhibited polygonal morphology, which is typical of epithelial cells. Furthermore, immunofluorescence revealed the expression of keratins 8 and 14, thereby confirming the epithelial origin of the cells. DNA content analysis of the three OSCC cell lines revealed aneuploidy. Furthermore, an in vitro orosphere assay revealed the formation of primary orospheres. Notably, the OSCC cell lines were able to give rise to tumors when administered subcutaneously into non-obese diabetic/severe combined immune deficiency mice. The novelty of the cell lines was also validated by performing short tandem repeat profiling; the STR profiles of the present cell lines did not significantly match with any known established OSCC cell lines present in the DSMZ database, thereby confirming the unique identity of these lines. These cell lines established from
\end{abstract}

Correspondence to: Dr Sanjeev K. Waghmare, Stem Cell Biology Group, Waghmare Lab, Cancer Research Institute, Advanced Centre for Treatment Research and Education in Cancer (ACTREC), Tata Memorial Centre, Sector 22, Utsav Chowk - CISF Road, Kharghar, Navi Mumbai 410210, India

E-mail: swaghmare@actrec.gov.in

*Contributed equally

Key words: oral squamous cell carcinoma, cell lines, orospheres, tumorigenicity tumor samples derived from Indian OSCC patients provide a valuable resource to understand the molecular mechanism involved in tumor resistance and recurrence.

\section{Introduction}

Head and neck squamous cell carcinoma (HNSCC) is the sixth most common cancer in the world. The annual global incidence of this disease exceeds 630,000 with an annual mortality of $\sim 350,000$ (1-4). Asia accounts for $57.5 \%$ of global HNSCC occurrence. In India, HNSCC is the most common cancer in males, which may be attributed to profound exposure of the population to lifestyle risk factors, including tobacco, cigarettes, areca nuts, and alcohol $(3,5,6)$. Recently, human papillomavirus (HPV) infection has also been suggested to be a risk factor for HNSCC $(3,5,6)$. In India, patients that are diagnosed at early and advanced stages of HNSCC have 5-year survival rates of 82 and $27 \%$, respectively (7).

A better understanding of molecular and biological characteristics of cancer cells may help in designing strategies for the prevention of or finding alternative drug targets for HNSCC treatment. Notably, the cell lines established from primary tumors retain the heterogeneity of the cell population as displayed by the parent tumors (8). Such characterization requires a constant and reliable source of cells belonging to a particular HNSCC. There have been a plethora of reports on the establishment of HNSCC cell lines. In the USA, initial attempts were made to establish HNSCC cell lines $(9,10)$; however, these cell lines were reported to be cross-contaminated with the HeLa cell line (11). Subsequently, in the UK, 10 HNSCC cell lines were established from tongue and larynx of patients who had undergone chemotherapy and radiotherapy. Among the 10 cell lines, 9 exhibited secretion of immune-reactive $\beta$ human chorionic gonadotropin (12). Another study demonstrated differences in the differentiation status, number of desmosomes, and expression of tonofilaments between primary and recurrent tumors (13). Heo et al established 21 HNSCC cell lines that were aneuploid and resistant to natural killer cells; however, these cell lines were 
efficiently lysed by lymphokine-activated killer cells (14). In the United States, establishment of 85 cell lines from Head and Neck tumor site was reported. The origins of these cell lines included head and neck squamous cell carcinoma, thyroid cancer, cutaneous squamous cell carcinoma, adenoid cystic carcinoma, oral leukoplakia, immortalized primary keratinocytes and normal epithelium (15). White et al (16) reported establishment of 52 HNSCC cell lines; the tendency of tumors with poor prognosis to successfully form cell lines was reported. In addition, HNSCC cell lines were able to form spheroids that exhibited a higher expression of the cancer stem cell (CSC) markers cluster of differentiation (CD) 44, CD133, sex determining region Y-box 2 and BMI1, as compared with normal epithelial oral cells (17). Recently, 16 cell lines were established from the tongue (10 cell lines), alveolus (4 cell lines), buccal mucosa (1 cell line) and hard palate (1 cell line) (18). Whole exome sequencing revealed upregulation of FAT1 and CASP8 in these cell lines as compared with blood samples of the matched patients. Furthermore, the individual or simultaneous knockdown of FATI and CASP8 genes exhibited reduced intercellular adhesion (18). Additionally, an oral tongue squamous cell carcinoma cell line was established from a non-smoker human papillomavirus (HPV)-negative patient (19). Whole exome sequencing of this cell line revealed mutations in the genes CDKN2A, TP53, SPTBN5, NOTCH2 and FAM136A (19).

In addition, two buccal mucosa carcinoma cell lines have been established from Chinese patients with one positive for HPV (20). Furthermore, a Japanese group have reported the establishment of 2 tongue-derived squamous carcinoma cell lines (one Node-positive and one Node-negative cell line). The node-positive cell line exhibited higher expression of keratins $8 / 18$ as compared with the node-negative cell line. Furthermore, the downregulation of keratins 13, 14, and 16 in these cell lines was associated with the invasive and metastatic abilities of cancer cells (21). Subsequently, 6 HNSCC cell lines were established in Korea. The study revealed that the cell lines exhibited a P53 transversion mutation in exon 7 and a transition mutation in the exon 8 (22). Furthermore, in Malaysia, cell lines were established from oral cavity cancers, which exhibited mutations in the P53 gene; one of the cell lines exhibited mouse double minute 2 homolog overexpression and the other exhibited epidermal growth factor receptor overexpression (23).

The establishment of HNSCC cell lines from Indian patients has previously been achieved from carcinomas of tongue, alveolus and retromolar trigone $(24,25)$; however, these cell lines did not possess the ability to give rise to tumors when injected into immune-compromised mice $(24,25)$. Furthermore, the establishment of a cell line from carcinoma of the upper aerodigestive tract, which can give rise to tumors when subcutaneously xenografted in nude mice was reported (26). In the present study, the establishment of three cell lines is reported, namely ACOSC3, ACOSC4 and ACOSC16, from advanced-stage treatment-naive squamous cell carcinomas of the buccal mucosa. The epithelial nature of the cell lines was confirmed by performing immunofluorescence assay (IFA) for keratins 8 and 14. The ploidy of the cell lines was assessed by flow cytometry and karyotyping. The novelty of the cell lines was also validated by determining their short tandem repeat (STR) profiles. These cell lines also formed orospheres (spheroids) when they were exposed to low-adherence conditions. The primary orospheres obtained from the cell lines also produced secondary orospheres. Notably, the cell lines were able to give rise to tumors when administered subcutaneously into non-obese diabetic/severe combined immune deficiency (NOD/SCID) mice.

\section{Materials and methods}

Patient sample collection and processing. A total of 16 advanced stage treatment naive oral squamous cell carcinoma (OSCC) samples were collected from patients at the department of Head and Neck Oncology, Tata Memorial Centre (Mumbai, India; TMH and ACTREC biorepositories) between May and October 2016. The ages of the patients ranged between 33 and 70 years. Of the 16 patients, 15 were males and 1 was female. Patients with human immunodeficiency virus or hepatitis B virus infections were excluded from the study. Fresh tumor samples were removed and collected by surgical resection from advanced-stage treatment-naive oral cancer and were stored in sterile vials. These samples were transported on ice and were treated with a $10 \%$ povidone-iodine solution (Wokadine ${ }^{\mathrm{TM}}$ ) for disinfection, followed by washing with sterile PBS to remove any traces of the disinfectant.

Explant culture. The samples were minced into fine pieces (2-3 $\mathrm{mm}$ ) by using surgical blades. These pieces were then placed on 35-mm (diameter) tissue culture plates (Thermo Fisher Scientific, Inc., Waltham, MA, USA) containing medium for explant culture. The medium contained minimum essential medium (MEM; Thermo Fisher Scientific, Inc.) supplemented with $10 \%$ horse serum (Thermo Fisher Scientific, Inc.) and $10 \%$ fetal bovine serum (HiMedia Laboratories, Mumbai, India). The explants were maintained at $37^{\circ} \mathrm{C}$ with $5 \% \mathrm{CO}_{2}$ atmosphere. The explant cultures were regularly monitored for cell growth. Excessive fibroblast growth was avoided by passaging with differential trypsinization, and keratinocytes were passaged when they reached $75-80 \%$ confluency.

Cell culture and storage. The cell lines were trypsinized using $0.25 \%$ trypsin (EDTA) with glucose. The cell monolayers were washed twice with PBS and trypsinized for 2-3 min. Trypsin was neutralized using complete medium. The cells were frozen and stored in liquid nitrogen in medium containing $10 \%$ dimethyl sulfoxide (DMSO). The vials containing the cell suspension and DMSO were cooled to $-80^{\circ} \mathrm{C}$ at a rate of $1^{\circ} \mathrm{C} /$ min prior to being transferred to liquid nitrogen.

Determination of doubling time. Cell lines were inoculated in 60-mm wide tissue culture plates (cat. no. 353002; Corning Incorporated, Corning, NY, USA). The cells were incubated at $37^{\circ} \mathrm{C}$ at $5 \% \mathrm{CO}_{2}$ atmosphere for up to $96 \mathrm{~h}$. The cells were counted at $24-\mathrm{h}$ intervals. Doubling time was calculated using the formula: $\mathrm{T} . \ln 2 / \ln (\mathrm{Xe} / \mathrm{Xb})$ where $\mathrm{T}=$ time of incubation, $\mathrm{Xe}=$ number of cells at the end of incubation time and $\mathrm{Xb}=$ number of cells at the beginning of the incubation time.

Immunocytochemical staining. Immunocytochemistry was performed on all three cell lines using primary antibodies 
against keratin 8 (1:100; cat. no. C5301; Sigma-Aldrich; Merck KGaA, Darmstadt, Germany) and keratin 14 (1:250; cat. no. MCA890; Serotech; Bio-Rad Laboratories, Inc., Hercules, CA, USA). Briefly, the cells were fixed at $-20^{\circ} \mathrm{C}$ for $20 \mathrm{~min}$ using absolute methanol and were permeabilized using a $0.3 \%$ solution of Triton in methanol. This was followed by blocking with $5 \%$ fetal bovine serum for $1 \mathrm{~h}$ at room temperature. Furthermore, the cells were incubated with the primary antibodies overnight at $4{ }^{\circ} \mathrm{C}$, followed by incubation with a fluorescein isothiocyanate-conjugated (1:400; cat. no. 115095003) and cyanine-3-conjugated (1:400; cat. no. 715165151; both Jackson ImmunoReseach Laboratories, Inc., West Grove, PA, USA) secondary antibody for $1 \mathrm{~h}$ at room temperature. Immunocytochemistry for CD44 was conducted using a mouse monoclonal antibody (1:600; cat. no. 156-3C11; Cell Signaling Technology, Inc., Danvers, MA, USA). The cells were fixed at room temperature with $4 \%$ formaldehyde for 20 min, permeabilized via $0.3 \%$ Triton X-100 in PBS, and blocked using 5\% normal goat serum (cat. no. 005000121; Jackson ImmunoReseach Laboratories, Inc.) for $1 \mathrm{~h}$ at room temperature. Following blocking, the cells were incubated with the primary antibody overnight at $4^{\circ} \mathrm{C}$. The cells were then treated with the cyanine-3-conjugated secondary antibody for $1 \mathrm{~h}$ at room temperature. The images were recorded using a confocal microscope at x400 magnification.

Determination of ploidy. Cells from all lines were harvested following trypsinization and resuspended in PBS containing RNase (cat. no. R5000; Sigma-Aldrich; Merck KGaA) $(0.2 \mathrm{mg} / \mathrm{ml})$ and propidium iodide $(0.08 \mathrm{mg} / \mathrm{ml})$ and incubated at $37^{\circ} \mathrm{C}$ for $30 \mathrm{~min}$ for DNA staining, and the DNA content was compared with that of human mononuclear cells from peripheral blood, (cat. no. 690PB-100A; Sigma-Aldrich; Merck KGaA), which served as a control for diploid human genomic DNA content. The cells were analyzed using a BD FACSCalibur system to determine their fluorescence. The data was analyzed by using ModFit 2.0 software (Verity Software House, Inc., Topsham, ME, USA). The mean channel of cells that were in the $\mathrm{G}_{0}$ phase was divided by that of the lymphocytes to determine DNA index of each cell line. The DNA index was then used to predict ploidy number of the cells.

HPV typing. DNA was extracted from each cell line using a lysis buffer containing $0.4 \mathrm{M}$ Tris ( $\mathrm{pH} 8.0$ ), $0.1 \mathrm{M} \mathrm{NaCl}$, $0.005 \mathrm{M}$ EDTA, $0.2 \%$ SDS and proteinase $\mathrm{K}$. The isolated DNA was screened for HPV infection by performing polymerase chain reaction (PCR), using a KAPA Taq PCR kit (cat. no. KK1015; Sigma-Aldrich; Merck KGaA) with MY09 (5'-CGTCCMARRGGAWACTGATC-3') and MY11 (5'-GCM CAGGGWCATAAYAATGG-3') primers (27). The following thermocycling conditions were used: Initial denaturation at $95^{\circ} \mathrm{C}$ for $5 \mathrm{~min}$ was followed by 40 cycles of denaturation at $95^{\circ} \mathrm{C}$ for $1 \mathrm{~min}$, annealing at $55^{\circ} \mathrm{C}$ for $1 \mathrm{~min}$ and elongation at $72^{\circ} \mathrm{C}$ for $1 \mathrm{~min}$. At the end of 40 cycles, the final elongation was carried out at $72^{\circ} \mathrm{C}$ for $10 \mathrm{~min}$. The MY09 and MY11 primers were used to amplify a 450-bp region in the HPV genome. DNA isolated from the HeLa cell line was obtained from Dr Manoj Mahimkar (ACTREC, Tata Memorial Center, Mumbai, India) to be used as the positive control for the HPV detection PCR. DNA isolated from MCF7 cell line (obtained from Dr Santosh Kumar, Bhaba Atomic Reseach Centre, Mumbai, India) was used as the negative control for the assay.

Karyotping of the cell lines. Chromosome analysis was performed by using a modification of the standard procedures used for karyotyping from fibroblast cultures (28). The metaphases tended to overspread. Therefore, the trypsinization time was reduced for G-banding. For cytogenetic analysis, 50 metaphase spreads were screened for ploidy in all three cell lines.

Orosphere formation assay. To evaluate the capability of the three oral cancer cell lines to produce spheroids in vitro, each cell line was subjected to the spheroid formation assay. In total, 5,000 cells were grown in complete MammoCult medium (MammoCult ${ }^{\mathrm{TM}}$ kit; basal human medium; cat. no. 05621; Stemcell Technologies, Inc., Vancouver, BC, Canada) in 6-well flat bottom ultra-low attachment plates (cat. no. 3471; Costar; Corning Incorporated) and were incubated at $37^{\circ} \mathrm{C}$ in a $\mathrm{CO}_{2}$ incubator. Primary orospheres were obtained following 3-4 days of culture; these primary orospheres were further trypsinized and subjected to secondary spheroid culture to assess the ability of cells to form secondary orospheres.

Tumorigenesis assay. To assess the tumorigenic potential of the three OSCC cell lines, 2 million cells of each cell line were resuspended in $200 \mu \mathrm{l}$ culture medium supplemented with $25 \%$ Matrigel (cat. no. 356230; Corning Incorporated) and were xenografted subcutaneously into the NOD/SCID mice. Animal protocols were approved by the Institutional Animal Ethics Committee Advanced Centre for Treatment, Education and Research in Cancer (ACTREC), Tata Memorial Centre (Navi Mumbai, India). NOD/SCID mice for tumorigenesis assay were procured from the Institutional Animal Facility at ACTREC. For the tumorigenesis assay, 15 female NOD/SCID mice with 5 replicates $(n=5)$ per group for ACOSC 3, ACOSC 4 and ACOSC16 were used. The mice were aged 6-8 weeks and weighed 20-22 g. The measurements of tumor diameters and volumes were recorded once per week using a Vernier caliper. Mice were sacrificed when the tumors volume reached a maximum diameter of $20 \mathrm{~mm}$. The dynamics of subcutaneous tumor growth were evaluated in ACOSC4 $(n=5)$ and ACOSC16 $(n=3)$ cell lines. The histograms of tumor volume were plotted by using GraphPad Prism version 5 software (GraphPad Software, Inc., La Jolla, CA, USA). Mice were maintained at a temperature of $22 \pm 2^{\circ} \mathrm{C}$, humidity of $50 \pm 5 \%$, and under positive air pressure with $12-15$ air changes/h. A light/dark cycle of 12-h was maintained. Mice were provided food and water ad libitum.

Cell line authentication by STR profiling. STR profiles of the cell lines were determined using 16 STR markers. The cells of the three OSCC cell lines were centrifuged at $162 \mathrm{xg}$ for $5 \mathrm{~min}$, and their DNA was extracted using GenElute ${ }^{\mathrm{TM}}$ Mammalian Genomic DNA Miniprep Kit (cat. no. G1N350; Sigma-Aldrich; Merck KGaA). To protect the identity of the patients, only eight of these markers have been provided in the data. Data analysis was performed using GeneMapper ${ }^{\circledR}$ ID-X software version 1.4 (Thermo Fisher Scientific, Inc.). The STR 
Table I. Clinical and pathological findings of established oral cancer cell lines.

\begin{tabular}{lcccccc}
\hline Cell line & $\begin{array}{c}\text { Patient } \\
\text { age (years) }\end{array}$ & Sex & Origin of tumor & $\begin{array}{c}\text { Pathological } \\
\text { staging (TNM) }\end{array}$ & Tumor diagnosis & Oral habit \\
\hline ACOSC3 & 40 & Male & Buccal mucosa & pT2 N2b M0 & $\begin{array}{l}\text { Squamous cell carcinoma; } \\
\text { moderately differentiated }\end{array}$ & Tobacco chewer \\
ACOSC4 & 70 & Female & Buccal mucosa & pT4 N2b M0 & $\begin{array}{l}\text { Squamous cell carcinoma; } \\
\text { moderately differentiated }\end{array}$ & Tobacco chewer \\
ACOSC16 & 34 & Male & Buccal mucosa & pT4a N2b M0 & $\begin{array}{l}\text { Squamous cell carcinoma; } \\
\text { moderately differentiated }\end{array}$ & Tobacco chewer \\
\hline
\end{tabular}

profiling was performed using PowerPlex ${ }^{\circledR} 16$ HS System (Promega Corporation, Madison, WI, USA). The profiles were compared to the DSMZ STR profiles database (29).

\section{Results}

Establishment of the cell lines from the advanced stage treatment naive oral cancer tissues. Tissue samples from advanced-stage treatment-naive patients with oral cancer were used for explant culture and establishing the cell lines (Table I). The processed tissue samples were inoculated in complete MEM, which was changed every 2 days. Over 2-3 weeks, the explants exhibited the growth of keratinocytes with a compact, clustered cell morphology and fibroblasts with an elongated morphology surrounding the explant tissues. The selective removal of the fibroblasts was performed by differential trypsinization to enrich the keratinocytes. During the initial passages, the growth of the cells was observed in discrete patches. Following several passages, the pure keratinocyte cultures were obtained from 3 different tissue samples, which were sub-cultured for $>40$ passages. Importantly, all three cell lines exhibited and maintained the polygonal cell shape, which is typical of epithelial cell morphology (Fig. 1). Furthermore, the rate of cell division of all three cell lines was measured by calculating their doubling time. The data indicated that the doubling times of the cell lines ACOSC3, ACOSC4, and ACOSC16 were 45.42, 33.78, and $34.00 \mathrm{~h}$ respectively. These results suggest that the established OSCC cell lines had long-term expansion potential and stable cell morphology.

Evaluation of the keratin and CD44 expression in the established cell lines. Epithelial cells derived from the different epithelial tissues displayed the distinct expression pattern of the cytokeratin isoforms. In addition, cancer cells originating from epithelial tissues exhibited abnormal expression of different cytokeratins. Immunocytochemistry was performed on the OSCC cell lines using the keratins 14 and 8, which are expressed in the basal cells of the epithelium and transformed keratinocytes, respectively $(30,31)$. The data indicated that the ACOSC4 cell line exhibited uniform and bright staining for keratin 14, whereas the cell lines ACOSC 3 and ACOSC16 exhibited heterogeneity in keratin 14 expression; certain cells exhibited bright staining for keratin 14, whereas other cells were completely negative for keratin 14 (Fig. 2A). In addition, keratin 8 expression analysis indicated that the three OSCC cell lines expressed different levels of keratin 8 . ACOSC 3 cells had the brightest staining for keratin 8, and ACOSC16 cells exhibited only slightly brighter staining for keratin 8 than ACOSC4 cells (Fig. 2B). IFA was performed using CD44 (CSC marker), which was expressed in all three cell lines (Fig. 2C). Collectively, these data suggest that all three OSCC cell lines are derived from basal keratinocytes (epithelial origin), have abnormal expression of the tumor-associated protein keratin 8 , and contain a CSC subpopulation.

Determination of ploidy. Abnormal DNA content in tumor cells due to defective mitosis or amplification of different DNA fragments is a leading hallmark of cancer. DNA content in all three OSCC cell lines was evaluated by performing ploidy analysis. Normal human lymphocytes were used as a control for diploid DNA content. The DNA index number was calculated by dividing mean channel for the cells in the $\mathrm{G}_{0}$ phase by that of the diploid lymphocytes. The DNA indices for the ACOSC 3 , ACOSC4, and ACOSC 16 cell lines were calculated to be $1.360,2.577$ and 2.377 , respectively (Fig. 3A-D). ACOSC3 cells had slightly higher than diploid DNA content, whereas both ACOSC4 and ACOSC16 cell lines exhibited slightly higher than tetraploid DNA content. These results indicated that all three cell lines have abnormal DNA content; which may be further investigated to identify the potential role of abnormal DNA content in the transformation of the cells.

HPV typing. Infection by HPV 16 and 18 has raised concern as a risk factor for oral cancers. Approximately $25 \%$ of oropharyngeal cancers are attributed to HPV 16 infection, whereas $1-3 \%$ of all oropharyngeal cancers are attributed to HPV 18 infection (32). HPV-positive cancers are associated with a more favorable prognosis as compared with HPV-negative cancers (33). To examine the HPV status in all three oral cancer cell lines, PCR was performed. DNA from the HeLa cell line was used as the positive control for HPV detection, whereas DNA isolated from the MCF7 cell line was used as the negative control. All three OSCC cell lines were found to be HPV negative by PCR using the MY09/MY11 primer sets (Fig. 3E).

Karyotyping of the cell lines. To precisely determine ploidy and gain insight into the chromosomal abnormalities in the 

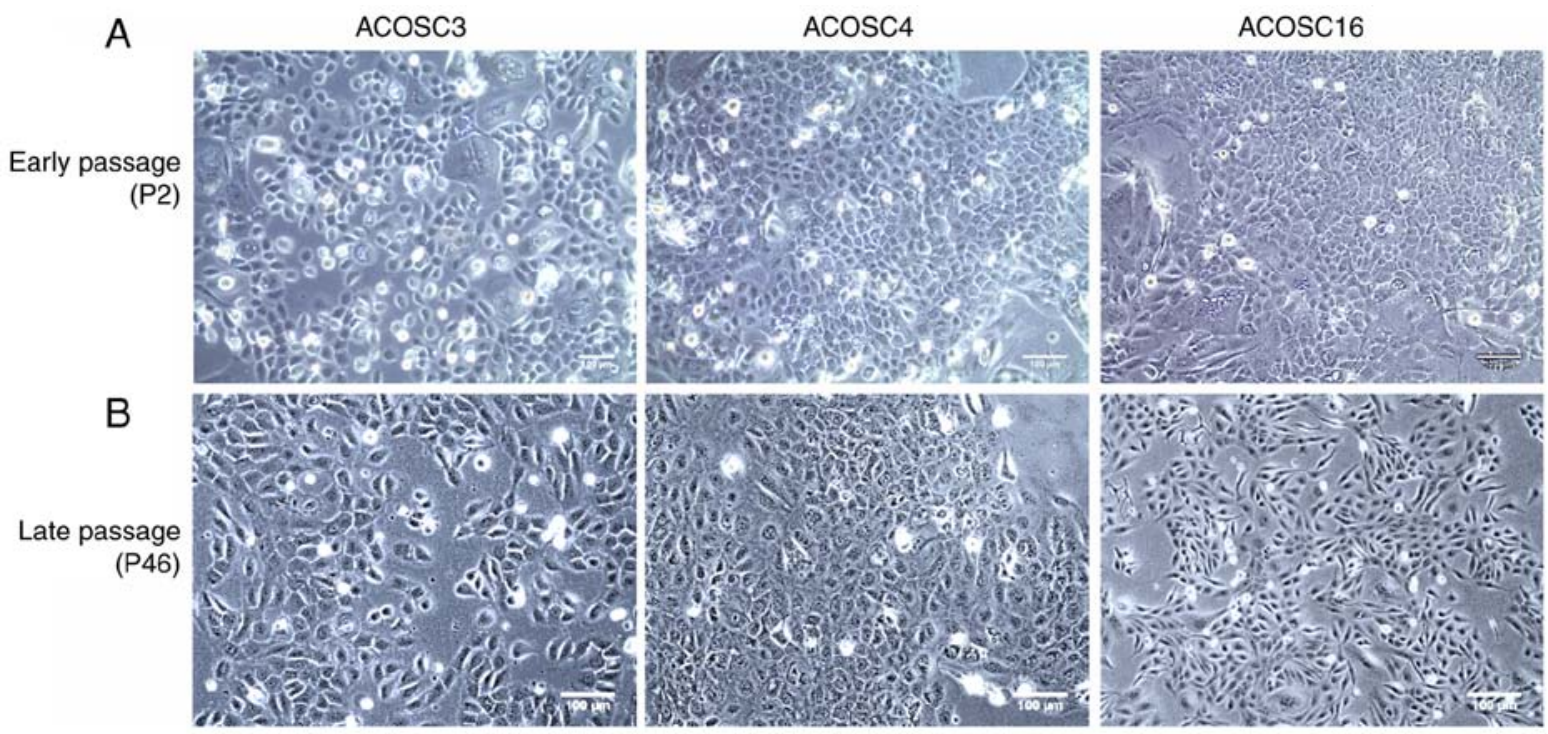

Figure 1. Morphological characteristics of established cell lines. All three cell lines (ACOSC3, ACOSC4 and ACOSC16) were cultured on 60-mm plates and observed under inverted microscopy. (A) Represents the cells of the early passage (P2). (B) Represents the cells of the late passage (P46). Scale bar, 100 $\mu \mathrm{m}$.

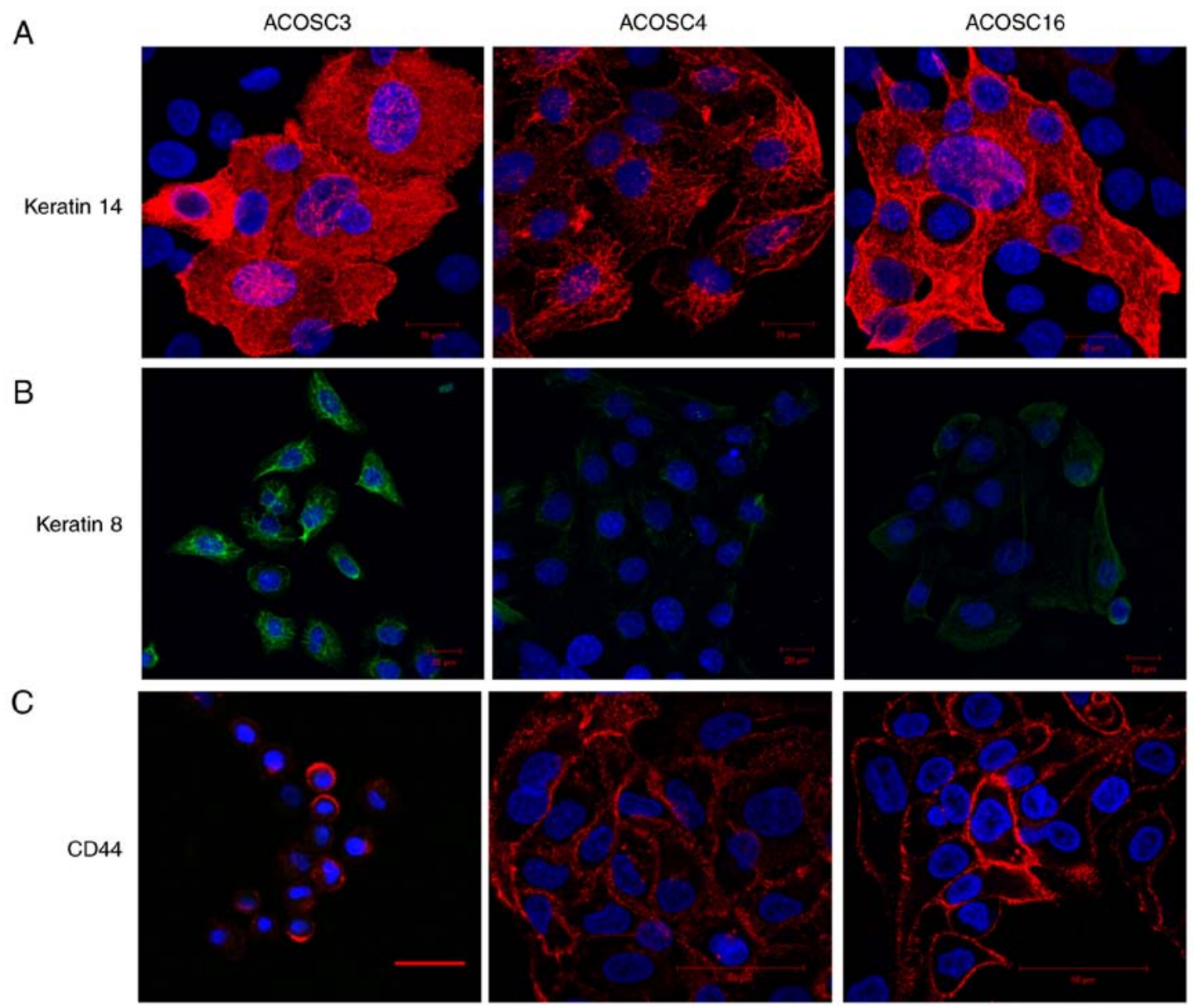

Figure 2. Immunofluorescence staining for epithelial markers. Cells of all three cell lines were analyzed for the expression of different epithelial markers. (A) Immunofluorescence staining of keratin 8. Scale bar, $20 \mu \mathrm{m}$. (B) Immunofluorescence staining of the basal keratin 14 . Scale bar, $20 \mu \mathrm{m}$. (C) Immunofluorescence staining of cancer stem cell marker CD44. DAPI was used to stain the nucleus, fluorescein isothiocyanate for keratin 8 (green), cyanine-3 for keratin 14 (red) and cyanaine-3 for CD44 (red). Scale bar, $50 \mu \mathrm{m}$. CD, cluster of differentiation.

cell lines, karyotyping of all three cell lines was performed. None of the cell lines exhibited metaphases with a normal karyotype (Fig. 4).
The ACOSC 3 cell line was aneuploid, with hyperdiploidy (64-80 chromosomes) and near-diploidy (40-41 chromosomes) in 70 and $30 \%$ of the metaphases, respectively. The loss of the 

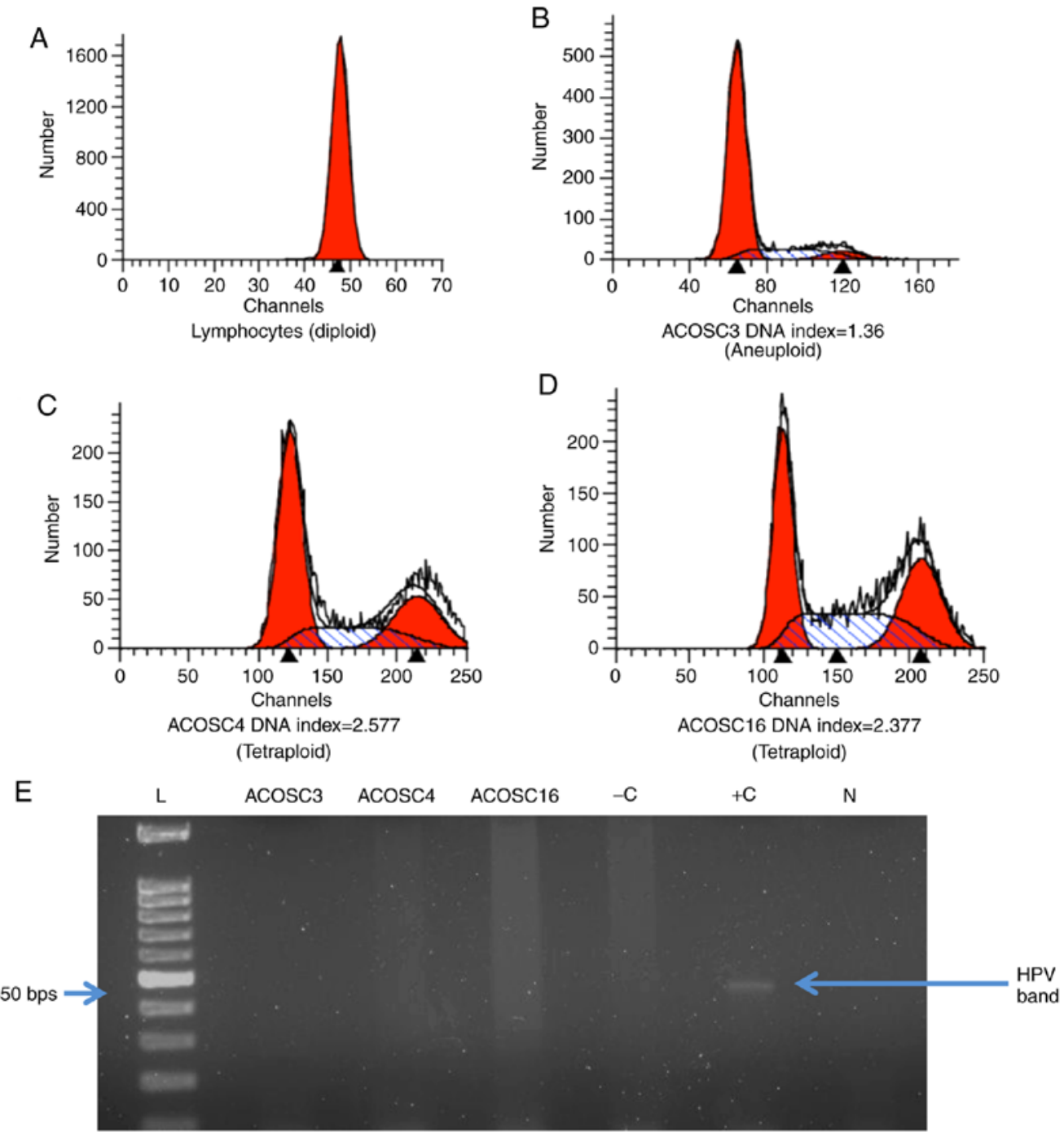

Figure 3. Flow cytometry analysis to assess the genomic DNA content. The genomic DNA content was analyzed by flow cytometry. (A) Normal human lymphocytes were used as a control for diploid DNA content, (B) ACOSC3 cell line with aneuploid genomic DNA content, (C) ACOSC4 cell line with tetraploid genomic DNA content and (D) ACOSC16 cell line with tetraploid genomic DNA content. (E) HPV undetected in all the oral cancer cell lines. Genomic DNA was isolated from all three cell lines and subjected to polymerase chain reaction analysis by using the MY09/MY11 primers specific for HPV capsid genes, having an expected band size of $450 \mathrm{bp}$. HPV, human papillomavirus; L, molecular marker; -C, negative control (MCF7 genomic DNA); +C, positive control [HeLa cell line infected with HPV (450 bp)]; N, non-template control.

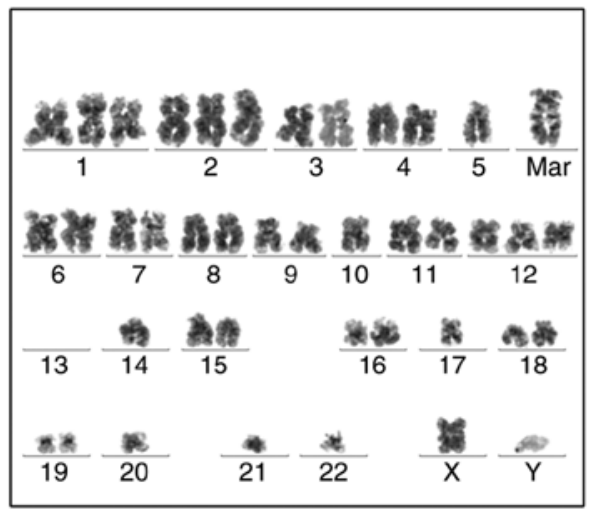

Karyotype of ACOSC3

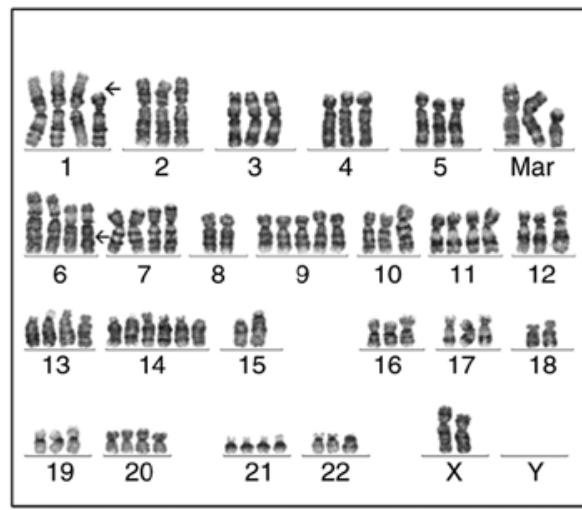

Karyotype of ACOSC4

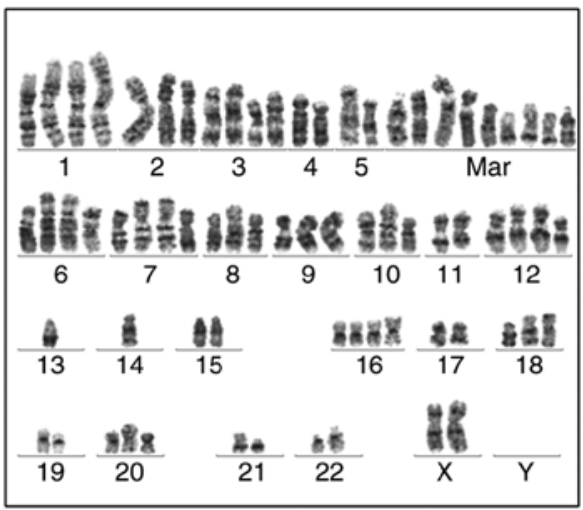

Karyotype of ACOSC16

Figure 4. Karyotyping of cell lines. All three cell lines exhibited abnormal karyotypes. Heterogeneity was observed in copy number of each chromosome in different metaphases of the same cell line. The cells exhibited random gains or losses of chromosomal regions as well as entire chromosomes. Notably, ACOSC 3 and ACOSC4 exhibited two subpopulations; namely, near diploid and near tetraploid. Certain markers which could not be identified as any of the normal chromosomes were also observed in the cell lines. ACOSC 3 had $70 \%$ near tetraploid cells and $30 \%$ near diploid cells. ACOSC4 had $85 \%$ near tetraploid subpopulation and 15\% near diploid subpopulation. ACOOSC16 had an entire population near tetraploid. Loss of identifiable Y chromosome was also observed in this cell line. 
A

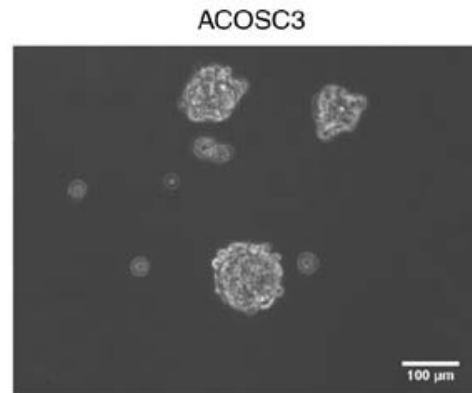

$\mathrm{B}$

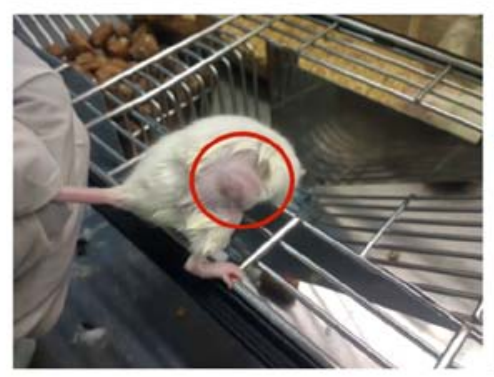

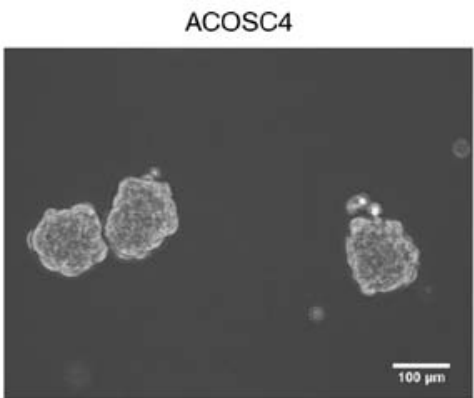

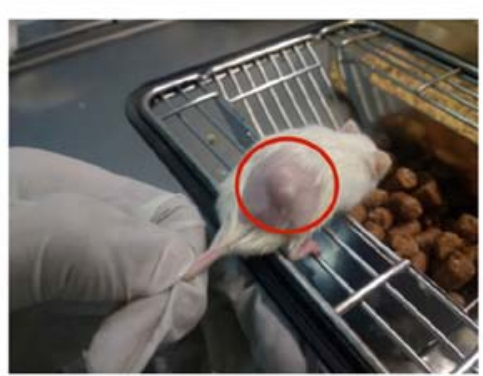

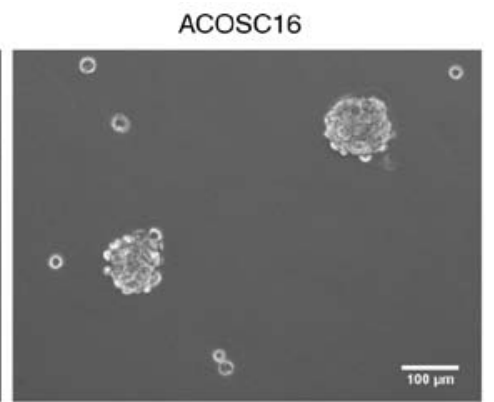

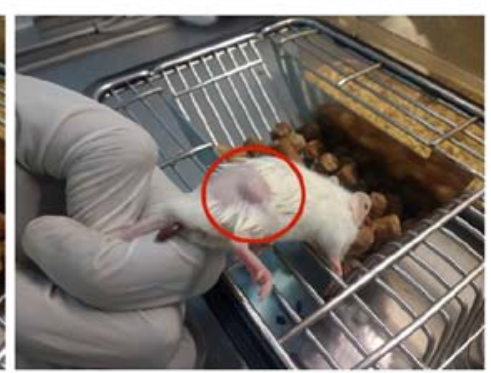

Figure 5. In vitro and in vivo characterization of cell lines. (A) Spheroid formation assay in the oral cancer cell lines: 5,000 cells from all three oral cancer cell lines (ACOSC3, ACOSC4 and ACOSC16) were seeded on 60-mm ultra-low attachment plates and were cultured for 5 days to monitor the spheroid formation. The images were captured by inverted microscopy. Scale bar, $100 \mu \mathrm{m}$. (B) In vivo tumorigenicity: $2 \times 10^{6}$ cells of each cell line (ACOSC3, ACOSC4 and ACOSC16) were administered subcutaneously in NOD/SCID mice, which were able to induce visible tumor within 2 weeks ( $=5$ ). Representative photos demonstrating established tumor following subcutaneous injection of cells in right side position (red circle) of NOD-SCID mice. NOD/SCID, non-obese diabetic/severe combined immune deficiency.

Y chromosome and two or three distinctive clonal markers was observed. One of these was a large submetacentric marker with sharp, equally spaced bands, which suggested amplification. Gains of chromosomes 1, 2, 3, 12, 19 and X were observed, whereas consistent losses of chromosomes 4, 7, 8, 13, 14, 21, 22 and $\mathrm{Y}$ were observed. A derivative chromosome 16 with very lightly stained short arms, suggesting t(16;19), was also observed. Seven metaphases were karyotyped.

The ACOSC4 cell line was aneuploid, with hyperdiploidy (63-83 chromosomes) in $85 \%$ of the metaphases and near-diploidy or pseudodiploidy in $\sim 15 \%$ of the metaphases. Clonal structural anomalies, including a derivative chromosome 1 with the loss of the 'p' arm and a probable unbalanced $\mathrm{t}(1 ; 12)(\mathrm{p} 11 ; \mathrm{p} 11)$, a suspicion of isochromosome 9q, deletion $6 \mathrm{q}$ and a Robertsonian $\mathrm{t}(15 ; 21)$ were detected. Five or six copies of chromosomes 9 and 14 were observed in some metaphases. Frequent losses of chromosomes 18 and 19 was also observed. Six metaphases were karyotyped.

ACOSC16 was aneuploid, with hyperdiploidy (55-71 chromosomes) and loss of the $\mathrm{Y}$ chromosome in all metaphases. Clonal structural anomalies included additional material on $1 \mathrm{p}$, deletion $3 \mathrm{p}$, isochromosome $9 \mathrm{q}$, additional material on $14 q 32$ (immunoglobulin heavy chain locus rearrangement) and 11q23 (MLL rearrangement) together with medium-sized markers. Loss of chromosomes 4, 5, 7, 8, 13, 17 and 21 was frequently observed. Five metaphases were karyotyped.

Orosphere formation assay. Cancer cells exhibit anchorage-independent cell growth. To evaluate the ability of these cells to form spheres in vitro, equal numbers $(10,000)$ of cells were cultured on a low-adherence plate for 5 days. The data suggested that the ACOSC3, ACOSC4, and ACOSC16 cell lines formed compactly rounded spheres, with high sphere-formation efficiency (Fig. 5A). Furthermore, these primary spheres were analyzed for their ability to give rise to secondary spheres. The primary spheres were trypsinized to prepare a single-cell suspension and were cultured in the low-adherence plate. Notably, when grown for 3-4 days, all three cell lines produced secondary orospheres. Collectively, these data suggest that the cells of all three OSCC cell lines are anchorage-independent, which is enhanced in stem cell populations.

Evaluation of the in vivo tumorigenic potential. Furthermore, to investigate the in vivo tumor formation efficiency of these cells, the NOD/SCID mice were subcutaneously injected with $2 \times 10^{6}$ cells of all three cell lines (ACOSC3, ACOSC4, and ACOSC16) with Matrigel. The results indicated that all three cell lines exhibited capability to give rise to tumors in immunocompromised mice. This experiment was performed in 5 replicates for each cell line, and all the mice injected with these cells exhibited tumor formation within 10-15 days. Each injection of the cells resulted in the formation of single, irregularly shaped tumors (Fig. 5B). Specifically, the ACOSC4 cell line was more aggressive as compared with ACOSC3 and ACOSC16 cell lines as it produced tumors in a relatively decreased duration of time (Fig. 6). These data confirm the tumorigenic potential of all three OSCC cell lines.

Cell line authentication through STR profiling. STR profiling was performed on all three OSCC cell lines to confirm that their novelty and genetic distinctness from previously established cell lines (Table II). The STR profiles of the three cell lines were compared with the DSMZ database. The profiles of the three cell lines did not exhibit any significant match with those of any previously established cell lines. 

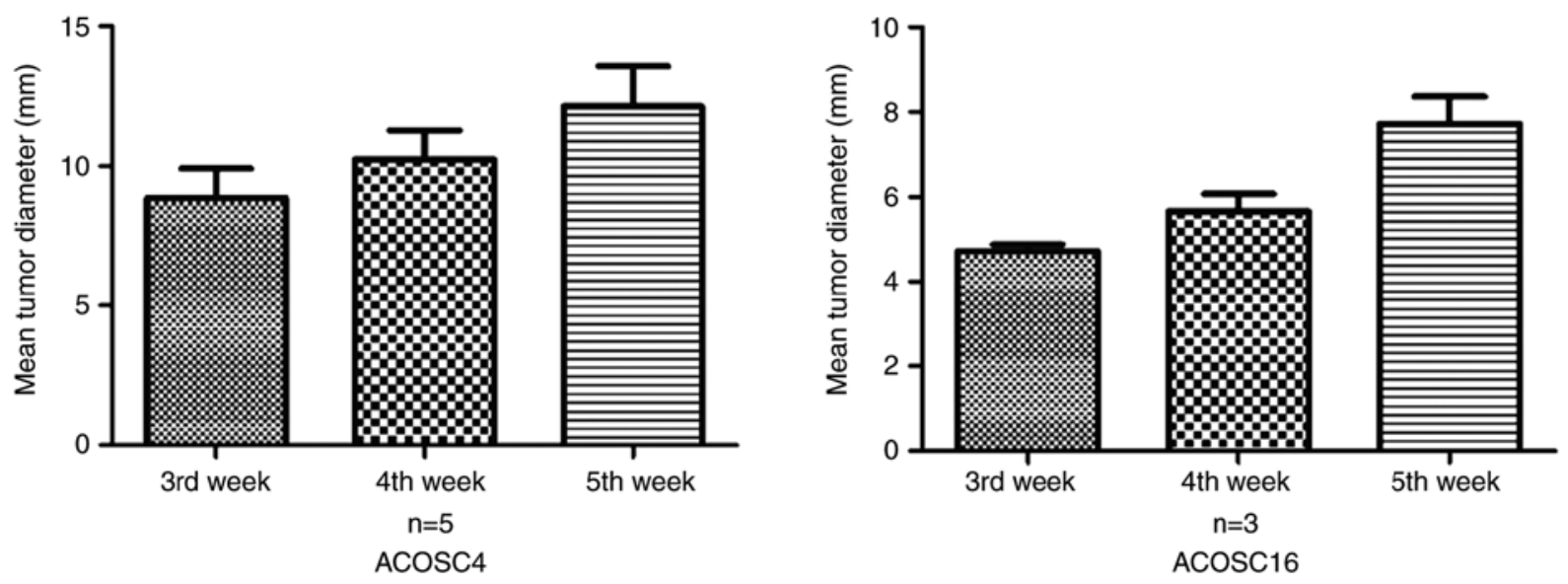

Figure 6. Dynamics of tumor growth. Cells of ACOSC4 and ACOSC16 cell lines were administered subcutaneously in 5 and 3 mice respectively. The number of the cells injected was $2 \times 10^{6}$. Growth of the tumors was monitored weekly. Mean diameters of tumors in 3rd, 4th, and 5th weeks have been plotted against mean tumor diameter.

Table II. Analysis of STR markers in all three cell lines.

\begin{tabular}{lccc}
\hline Marker & ACOSC3 & ACOSC4 & ACOSC16 \\
\hline TH01 & 6 & 6,8 & $7,9.3$ \\
D5S818 & 12,13 & 10,12 & 11 \\
D13S317 & 8,11 & 8,11 & 11 \\
D7S820 & 9,10 & 11 & 7,12 \\
D16S539 & 11 & 11,12 & 9,10 \\
CSF1PO & 10,12 & 10,11 & 11,12 \\
vWA & 14,15 & 16,29 & 15,29 \\
TPOX & 8,9 & 10,11 & 8 \\
Amel & $\mathrm{X}, \mathrm{Y}$ & $\mathrm{X}, \mathrm{X}$ & $\mathrm{X}, \mathrm{Y}$ \\
\hline
\end{tabular}

STR, short tandem repeat.

The STR profiles of the ACOSC3, ACOSC4 and ACOSC16 cell lines were distinct, thus confirming their uniqueness and the absence of cross-contamination. Collectively, these data confirm the novelty of the cell lines and that they are derived from the previously unreported tissue samples. It was confirmed that the cell lines were derived from the tumors donated by patients by determining the STR profiles of the tumors and matching them with the profiles of their respective cell lines.

\section{Discussion}

OSCC is the second most common cancer in India $(3,5,6)$. However, only a few cell lines of OSCC have been derived from Indian patients. Buccal mucosa carcinoma-derived cell lines were previously established using tissue samples from Indian patients could not form tumors when injected into immunocompromised mice (24). The establishment of cell lines from patients with oral cancers is crucial because studies on these cell lines have contributed considerably to the current understanding of tumor heterogeneity, and highlight any differences between the Indian (Asian) and
Western populations at a molecular level. OSCC presents a wide variation in epidemiology in populations from Western countries and Southeast Asian regions, which may be due to genetic differences between these populations (4). The cell lines established from patients with OSCC can provide a source of cancer cells that retain the heterogeneity of the cell populations present in the parental tumor. Therefore, they can be used as model systems to study pharmacokinetics and pharmacogenomics of newly discovered anticancer drugs. Notably, they can be used to understand the molecular mechanism involved in the resistance of cancer cells to chemotherapy and radiotherapy.

Recently, 16 HNSCC cell lines were established by using oral mucosa cancer tissue samples, and whole exome sequencing was performed along with patient-matched blood (18). The present study intended to identify the mutations present in the tumor and distinguish them from the mutations accumulated during sub-culturing the cell lines. A similar study on cell lines derived from Indian patients may reveal considerable information on cancer development and mode of action of the carcinogens frequently encountered by Indian patients, including tobacco, betel quid and areca nut.

A previous study demonstrated that cancer cells possess stem-like characteristics. These CSCs can be enriched the spheroid formation assay. Furthermore, $\mathrm{CD} 44^{+} / \mathrm{CD} 66^{-}$is a reliable combination of markers for the isolation of CSCs (17). Similarly, the presently established OSCC cell lines can also be used to identify a combination of markers for the identification of true CSCs in Indian patients, which can be useful in developing effective therapeutic strategies for these patients. CSCs can be isolated from the OSCC cell lines, and the molecular validation of the genes specifically deregulated in the CSCs can be performed.

The cell lines were established from treatment-naive advanced-stage cancer patients directly by using primary tumors, thus eliminating any genotypic or phenotypic changes arising because of patient-derived xenograft generation. These cell lines grow in an anchorage-independent manner and do not require any feeder cells to grow. The polygonal shape of the cells and their tendency to grow in discrete patches confirm 
the epithelial nature of the cell lines. The evidence of keratins 8 and 14 expression additionally supports this inference as keratins are exclusively expressed by epithelial cells (34).

DNA ploidy analysis of cancer cells can provide an understanding of the aggressiveness, metastatic potential, and prognosis of the disease (35-37). The DNA content of all three cell lines was higher than the diploid DNA content of the lymphocytes. The DNA index of ACOSC3 (1.36) implied hyperdiploid DNA content, whereas the DNA indices of ACOSC4 (2.577) and ACOSC16 (2.377) implied that the DNA content exceeded the tetraploid DNA content. This may be explained by the rapid and frequent divisions of cancer cells. Uncontrolled cell divisions result in improper karyokinesis, which results in an increase in the ploidy level of the cells (38).

Chromosomal analysis suggested that all three cell lines had abnormal karyotypes. In the ACOSC 3 cell line, two subpopulations were present; the larger subpopulation (70\%) was hyperdiploid (64-80 chromosomes), whereas the other $30 \%$ metaphases were near-diploid (40-41 chromosomes). In the ACOSC4 cell line, a hyperdiploid (63-83 chromosomes) subpopulation was observed, which constituted $85 \%$ of the karyotyped metaphases, whereas $\sim 15 \%$ of the metaphases were near-diploid or pseudodiploid. All 50 analyzed metaphases of the ACOSC16 cell line were hyperdiploid (55-71 chromosomes). All three cell lines exhibited gains, losses, and translocations of various chromosomal regions or entire chromosomes. These results underline the genomic instability of these cells.

HPV (high-risk HPV genotypes 16, 18, 31, 33, and 35) is one of the dominant risk factors for the development of OSCC. Evidence from the literature suggests that HPV infection is a risk factor for oral cancer (32). The present cell lines were evaluated for the presence of HPV markers by performing PCR using MY09/MY11 primers for the capsid protein of the virus. The present results indicated that all three of the cell lines were found to be free of HPV infection.

One of the notable properties of cancer stem cells $\left(\mathrm{CD}_{4} 4^{+} / \mathrm{ALDH}^{+}\right.$cells) is the formation of spheres when cultured on low-adherence plates (39). An orosphere assay was performed on all three cell lines to detect the presence of anchorage-independent CSCs, which were plated onto low-adherence plates; this confirmed the presence of CSCs within these cell lines. Additionally, immunofluorescence exhibited the expression of CD44, which is one of the CSC markers.

In vivo tumorigenesis assay is the gold standard assay to detect the presence of CSCs from a heterogeneous cell population. Tumorigenicity is a very important property of the cell lines as this property enables in-depth studies on the development of tumors and the development of therapy resistance in them to be conducted. Furthermore, all three cell lines produced tumors when injected into NOD/SCID mice. These cell lines can be used to generate xenografts, which can be used as model systems for testing effectiveness of therapeutic agents on tumors of human origin.

The novelty of the present OSCC cell lines was confirmed through STR profiling. The STR profiles of the three cell lines were compared with the DSMZ database of STR profiles. The STR profiles of the present cell lines did not significantly match with any of the cell lines present in the STR profile database or amongst each other. Also, the cell lines had differences in their growth patterns, keratin expression patterns and DNA content and STR profiles, thus confirming their unique nature (40).

Recently, a number of studies have been carried out in Western populations by using patient-derived tumor cell lines, which include exome sequencing that reveals a large amount of information regarding the mutations in several genes across the genome $(16,18,19)$. These studies enable deduction of molecular dysfunctions that may be associated with carcinogenesis and therapy resistance. These cell lines allow for extensive studies using cells from Indian patients. The cell lines may be used to further delineate the signaling networks, genetic, epigenetic, transcriptomic and proteomic characterization of the oral cancer cells derived from Indian patients, contributing towards a better understanding of the biology.

In conclusion, the present study reports the establishment of three novel OSCC cell lines from the advanced-stage treatment-naive oral cancer derived from buccal mucosa carcinoma patients of Indian ethnicity; all of them with a habit of tobacco consumption. These cell lines have a wide range of potential application for basic and translational research in oral cancer.

\section{Acknowledgements}

The authors would like to thank Ms. Sayoni Roy for helping in formatting the manuscript and Mr. Raghava R. Sunkara for his help in the discussion. We also thank Dr Prochi Madon (Department of Assisted Reproduction and Genetics, Jaslok Hospital and Research Centre, Mumbai, India) for her help in the karyotype determination and interpretation for the present study.

\section{Funding}

SSN is supported by CSIR fellowship. GLC is supported by ACTREC fellowship. The present study was supported from the grant of ACTREC-TMC intramural fund (grant no. 3542).

\section{Availability of data and materials}

All data generated and/or analyzed during this study are included in this published article.

\section{Authors' contributions}

SKW conceived and designed the study, and analyzed and interpreted the data. NPG and SSN performed the experiments. PC provided the tumor samples, and analyzed and interpreted the data. GLC analyzed and interpreted the data. SSN, GLC and SKW wrote the manuscript. NPG and SSN prepared the images. SKW and GLC reviewed the data. SKW reviewed the manuscript. All authors read and approved the final manuscript.

\section{Ethics approval and consent to participate}

The present study was approved by the Institutional Ethics Committee of Advanced Centre for Treatment, Education and Research in Cancer, Tata Memorial Centre (Navi Mumbai, India). Informed consent for the study was obtained from all the patients involved in the present study. 


\section{Patient consent for publication}

Informed consent for the study was obtained from all the patients involved in the study.

\section{Competing interests}

The authors declare that they have no competing interests.

\section{References}

1. Jemal A, Bray F, Center MM, Ferlay J, Ward E and Forman D: Global cancer statistics. CA Cancer J Clin 61: 69-90, 2011.

2. Jemal A, Siegel R, Ward E, Murray T, Xu J and Thun MJ: Cancer statistics, 2007. CA Cancer J Clin 57: 43-66, 2007.

3. Parkin DM, Bray F, Ferlay J and Pisani P: Global cancer statistics, 2002. CA Cancer J Clin 55: 74-108, 2005.

4. Vigneswaran $\mathrm{N}$ and Williams MD: Epidemiologic trends in head and neck cancer and aids in diagnosis. Oral Maxillofac Surg Clin North Am 26: 123-141, 2014.

5. Kulkarni M: Head and neck cancer burden in India. Int J Head Neck Surg 4: 29-35, 2013.

6. Torre LA, Bray F, Siegel RL, Ferlay J, Lortet-Tieulent J and Jemal A: Global cancer statistics, 2012. CA Cancer J Clin 65 87-108, 2015

7. Iype EM, Pandey M, Mathew A, Thomas G, Sebastian P and Nair MK: Oral cancer among patients under the age of 35 years. J Postgrad Med 47: 171-176, 2001

8. Beck B and Blanpain C: Unravelling cancer stem cell potential. Nat Rev Cancer 13: 727-738, 2013.

9. Eagle H: Propagation in a fluid medium of a human epidermoid carcinoma, strain KB. Proc Soc Exp Biol Med 89: 362-364, 1955.

10. Lin CJ, Grandis JR, Carey TE, Gollin SM, Whiteside TL, Koch WM, Ferris RL and Lai SY: Head and neck squamous cell carcinoma cell lines: Established models and rationale for selection. Head Neck 29: 163-188, 2007.

11. Lacroix M: Persistent use of 'false' cell lines. Int J Cancer 122 $1-4,2008$

12. Easty DM, Easty GC, Carter RL, Monaghan P and Butler LJ: Ten human carcinoma cell lines derived from squamous carcinomas of the head and neck. Br J Cancer 43: 772-785, 1981

13. Easty DM, Easty GC, Carter RL, Monaghan P, Pittam MR and James T: Five human tumour cell lines derived from a primary squamous carcinoma of the tongue, two subsequent local recurrences and two nodal metastases. Br J Cancer 44: 363-370, 1981

14. Heo DS, Snyderman C, Gollin SM, Pan S, Walker E, Deka R, Barnes EL, Johnson JT, Herberman RB and Whiteside TL: Biology, cytogenetics, and sensitivity to immunological effector cells of new head and neck squamous cell carcinoma lines. Cancer Res 49: 5167-5175, 1989.

15. Zhao M, Sano D, Pickering CR, Jasser SA, Henderson YC, Clayman GL, Sturgis EM, Ow TJ, Lotan R, Carey TE, et al: Assembly and initial characterization of a panel of 85 genomically validated cell lines from diverse head and neck tumor sites. Clin Cancer Res 17: 7248-7264, 2011.

16. White JS, Weissfeld JL, Ragin CC, Rossie KM, Martin CL, Shuster M, Ishwad CS, Law JC, Myers EN, Johnson JT and Gollin SM: The influence of clinical and demographic risk factors on the establishment of head and neck squamous cell carcinoma cell lines. Oral Oncol 43: 701-712, 2007.

17. Kaseb HO, Fohrer-Ting H, Lewis DW, Lagasse E and Gollin SM Identification, expansion and characterization of cancer cells with stem cell properties from head and neck squamous cell carcinomas. Exp Cell Res 348: 75-86, 2016.

18. Hayes TF, Benaich N, Goldie SJ, Sipilä K, Ames-Draycott A, Cai W, Yin G and Watt FM: Integrative genomic and functional analysis of human oral squamous cell carcinoma cell lines reveals synergistic effects of FAT1 and CASP8 inactivation. Cancer Lett 383: 106-114, 2016.

19. Wang SJ, Asthana S, van Zante A, Heaton CM, Phuchareon J, Stein L, Higuchi S, Kishimoto T, Chiu CY, Olshen AB, et al Establishment and characterization of an oral tongue squamous cell carcinoma cell line from a never-smoking patient. Oral Oncol 69: 1-10, 2017.
20. Wong DY, Chang KW, Chen CF and Chang RC: Characterization of two new cell lines derived from oral cavity human squamous cell carcinomas-OC1 and OC2. J Oral Maxillofac Surg 48: 385-390, 1990

21. Morifuji M, Taniguchi S, Sakai H, Nakabeppu Y and Ohishi M Differential expression of cytokeratin after orthotopic implantation of newly established human tongue cancer cell lines of defined metastatic ability. Am J Pathol 156: 1317-1326, 2000.

22. Lee EJ, Kim J, Lee SA, Kim EJ, Chun YC, Ryu MH and Yook JI: Characterization of newly established oral cancer cell lines derived from six squamous cell carcinoma and two mucoepidermoid carcinoma cells. Exp Mol Med 37: 379-390, 2005.

23. Hamid S, Lim KP, Zain RB, Ismail SM, Lau SH, Mustafa WM Abraham MT, Nam NA, Teo SH and Cheong SC: Establishment and characterization of Asian oral cancer cell lines as in vitro models to study a disease prevalent in Asia. Int J Mol Med 19: 453-460, 2007.

24. Tatake RJ, Rajaram N, Damle RN, Balsara B, Bhisey AN and Gangal SG: Establishment and characterization of four new squamous cell carcinoma cell lines derived from oral tumors. J Cancer Res Clin Oncol 116: 179-186, 1990.

25. Patil TT, Kowtal PK, Nikam A, Barkume MS, Patil A, Kane SV, Juvekar AS, Mahimkar MB and Kayal JJ: Establishment of a tongue squamous cell carcinoma cell line from indian gutka chewer. J Oral Oncol 2014: 9, 2014.

26. Mulherkar R, Goud AP, Wagle AS, Naresh KN, Mahimkar MB, Thomas SM, Pradhan SA and Deo MG: Establishment of a human squamous cell carcinoma cell line of the upper aero-digestive tract. Cancer Lett 118: 115-121, 1997.

27. Gravitt PE, Peyton CL, Alessi TQ, Wheeler CM, Coutlée F, Hildesheim A, Schiffman MH, Scott DR and Apple RJ: Improved amplification of genital human papillomaviruses. J Clin Microbiol 38: 357-361, 2000.

28. Rooney DE (ed): Human Cytogenetics: Constitutional Analysis, A Practical Approach. Oxford University Press, Oxford, 2001.

29. Dirks WG, MacLeod RA, Nakamura Y, Kohara A, Reid Y, Milch H, Drexler HG and Mizusawa H: Cell line cross-contamination initiative: An interactive reference database of STR profiles covering common cancer cell lines. Int J Cancer 126 : 303-304, 2010.

30. Coulombe PA, Kopan R and Fuchs E: Expression of keratin K14 in the epidermis and hair follicle: Insights into complex programs of differentiation. J Cell Biol 109: 2295-2312, 1989.

31. Matthias C, Mack B, Berghaus A and Gires O: Keratin 8 expression in head and neck epithelia. BMC Cancer 8: 267, 2008.

32. Ghittoni R, Accardi R, Chiocca S and Tommasino M: Role of human papillomaviruses in carcinogenesis. Ecancermedicalscience 9: 526, 2015.

33. Nagel R, Martens-de Kemp SR, Buijze M, Jacobs G, Braakhuis BJ and Brakenhoff RH: Treatment response of HPV-positive and HPV-negative head and neck squamous cell carcinoma cell lines. Oral Oncol 49: 560-566, 2013.

34. Herrmann H, Bar H, Kreplak L, Strelkov SV and Aebi U: Intermediate filaments: From cell architecture to nanomechanics. Nat Rev Mol Cell Biol 8: 562-573, 2007.

35. Stell PM: Ploidy in head and neck cancer: A review and meta-analysis. Clin Otolaryngol Allied Sci 16: 510-516, 1991.

36. Cooke LD, Cooke TG, Bootz F, Forster G, Helliwell TR, Spiller D and Stell PM: Ploidy as a prognostic indicator in end stage squamous cell carcinoma of the head and neck region treated with cisplatinum. Br J Cancer 61: 759-762, 1990.

37. Slootweg PJ, Rutgers DH and Wils IS: DNA ploidy analysis of squamous cell head and neck cancer to identify distant metastasis from second primary. Head Neck 14: 464-466, 1992.

38. Vitale I, Galluzzi L, Senovilla L, Criollo A, Jemaà M, Castedo M and Kroemer G: Illicit survival of cancer cells during polyploidization and depolyploidization. Cell Death Differ 18: 1403-1413, 2011.

39. Krishnamurthy S and Nor JE: Orosphere assay: A method for propagation of head and neck cancer stem cells. Head Neck 35: 1015-1021, 2013.

40. Parson W, Kirchebner R, Muhlmann R, Renner K, Kofler A, Schmidt $S$ and Kofler R: Cancer cell line identification by short tandem repeat profiling: Power and limitations. FASEB J 19: 434-436, 2005. 